\title{
Effects of general and spinal anesthetic techniques on endothelial adhesion molecules in cesarean section
}

\author{
Mehtap Honca ${ }^{1}$, Tarık Purtuloglu², Emin Ozgur Akgul ${ }^{3}$, Muzaffer Oztosun ${ }^{4}$, Tevfik Honca ${ }^{3}$, \\ Ali Sizlan ${ }^{2}$, Mehmet Agilli ${ }^{3}$ İbrahim Aydin ${ }^{3}$, Memduh Yetim², Fevzi Nuri Aydin ${ }^{3}$, and Halil Yaman ${ }^{3}$ \\ ${ }^{1}$ Department of Anesthesiology and Reanimation, Kecioren Training and Research Hospital, ${ }^{2}$ Department of Anesthesiology and \\ Reanimation, Gulhane Military Medical Academy, ${ }^{3}$ Department of Medical Biochemistry, Gulhane Military Medical Academy, \\ ${ }^{4}$ Department of Biochemistry, Turkish Armed Forces, Health Services Command, Etimesgut, Ankara, Turkey
}

Background: The aim of this study was to investigate the effects of anesthetic techniques used during general anesthesia (GA) and spinal anesthesia (SA) on endothelial adhesion molecules in the fetal circulation of healthy parturients undergoing elective cesarean section.

Methods: Patients were randomly assigned to either the general anesthesia $(n=20)$ or spinal anesthesia $(n=20)$ group. Maternal and cord blood neopterin, sE-selectin, and sL-selectin levels were measured in both groups.

Results: Cord blood neopterin concentrations in the SA group were not different from those in the GA group, but maternal neopterin levels in the SA group were different from those in the GA group. Maternal blood levels of sE-selectin and sL-selectin were not different between the two groups. Similarly, the cord blood levels of sE-selectin and sL-selectin were not different between the two groups. We found an increased inflammatory process in the fetal circulation depending on the anesthetic method used.

Conclusions: These results indicate the effects of general and spinal anesthetic techniques on serum sL-selectin, sEselectin, and neopterin levels in neonates and parturients undergoing elective cesarean section. sE-selectin and neopterin concentrations and leukocyte counts were higher in the fetal circulation than in the maternal circulation during both GA and SA. (Korean J Anesthesiol 2014; 66: 364-370)

Key Words: Cesarean section, Endothelial adhesion molecules, General anesthesia, Spinal anesthesia.

Received: September 10, 2013. Revised: October 21, 2013. Accepted: October 29, 2013.

Corresponding author: Mehtap Honca, M.D., Department of Anesthesiology and Reanimation, Kecioren Training and Research Hospital, Sanatoryum cad, Pinarbasi mah, Ardahan sok, No:25, Kecioren, Ankara 06380, Turkey. Tel: 90-312-3569000, Fax: 90-312-3569002, E-mail: mehtaphonca@hotmail.com

(c) This is an open-access article distributed under the terms of the Creative Commons Attribution Non-Commercial License (http:// creativecommons.org/licenses/by-nc/3.0/), which permits unrestricted non-commercial use, distribution, and reproduction in any medium, provided the original work is properly cited. 


\section{Introduction}

Spinal anesthesia (SA) is commonly preferred by anesthesiologists and gynecologists because of its beneficial effects for both mothers and their fetuses during elective cesarean section (CS). However, when SA is contraindicated, general anesthesia (GA) may be preferred [1]. During and after surgery, different methods of anesthesia and levels of surgical trauma cause metabolic and inflammatory changes that correlate with the extent of tissue damage [2]. The local and systemic inflammatory responses due to biologic stress during GA are significant and may be detrimental [3]. The sympathetic and somatic blockade of SA may prevent or inhibit inflammatory stress [4]. However, GA may or may not augment this response [5]. Activation of the neuroendocrine system is related to the inflammatory stress response, leukocyte activation, and the cytokine network during and after surgery [6]. In contrast, neuronal afferent stimuli cause the release of cytokines from damaged tissue and the activation of cellular and humoral immune pathways [7]. The anesthetic method may influence the cytokine response by direct pharmacological action or effects on nervous and hormonal pathways [2].

In addition to the activation of leukocytes, cell adhesion molecules such as selectins play an important role in the interaction between leukocytes and endothelial cells during the inflammatory process [8]. E-selectin and L-selectin are cell adhesion molecules [9]. E-selectin is expressed by activated endothelial cells, while L-selectin is expressed on the surface of most leukocytes. However, soluble forms of these selectins can be used as activation markers of endothelial cells (soluble E-selectin) and leukocytes (soluble L-selectin) [10]. Furthermore, these selectins have important roles in the regulation of leukocyte attachment and support of leukocyte rolling [9].

It has been shown that upregulation of proinflammatory cytokines such as interferon- $\gamma$ (INF- $\gamma$ ) and tumor necrosis factor- $\alpha$ is associated with infiltration of leukocytes to the maternal-fetal interface in the inflammatory process [11]. Neopterin is synthesized from guanosine triphosphate in macrophages and monocytes, when guanosine triphosphate cyclohydrolase I is activated by IFN- $\gamma$ and other cytokines [12]. It is a simple, reliable, and sensitive parameter of cell-mediated immunity, and it has been accepted as a strong indicator of the clinical severity of many diseases [13,14].

To our knowledge, no studies have shown the effects of GA and SA on the levels of cell adhesion molecules. The aim of this study was to determine the levels of these molecules in the fetal circulation of healthy parturients undergoing elective CS under GA and SA. We also aimed to evaluate a possible relationship between maternal and fetal inflammatory modifications. For this reason, we evaluated the effects of SA and GA on the levels of neopterin, sE-selectin, and sL-selectin in maternal and cord blood samples.

\section{Materials and Methods}

This study was carried out after approval from the Institutional Ethics Committee of Gulhane Military Medical Academy, Ankara, Turkey, and written informed consent was obtained from all subjects. According to the American Society of Anesthesiologists (ASA) Classification, 40 ASA I (normal, healthy) parturients scheduled for uncomplicated elective cesarean delivery at a gestational age of $>37$ weeks were included in this study. Patients with medical problems such as hypertension, diabetes mellitus, heart disease, a body mass index of $>35 \mathrm{~kg} / \mathrm{m}^{2}$, and a Mallampati score of $>2$ were not included in this study. Patients with any contraindication to either GA or SA were also excluded from the study.

All patients were randomly assigned to either the GA $(n=20)$ or SA $(n=20)$ group. After positioning the operation table with a $15^{\circ}$ left lateral tilt in the operating room, the patients were monitored with devices including automated blood pressure (cuff), electrocardiography, and pulse oximetry.

Before the operation, all patients in both groups received 500 $\mathrm{ml}$ of colloid solution (5\% Hetastarch) and $500 \mathrm{ml}$ of Ringer's lactate solution. After preoxygenation with $100 \%$ oxygen for $3 \mathrm{~min}$, thiopental $(4 \mathrm{mg} / \mathrm{kg})$ and rocuronium $(0.6-0.8 \mathrm{mg} / \mathrm{kg}$ ) were administered in the GA group. After endotracheal intubation, patients were ventilated to a target end-tidal carbon dioxide concentration of 30 to $35 \mathrm{mmHg}$. Anesthesia was maintained at an end-tidal concentration of $1 \%$ sevoflurane and $50 \%$ nitrous oxide in oxygen. Fentanyl $(1 \mu \mathrm{g} / \mathrm{kg})$ was administered after delivery and umbilical cord clamping. After delivery of the placenta, 10 IU of oxytocin were administered slowly via an intravenous route. A second continuous infusion of $15 \mathrm{IU}$ of oxytocin was given in $1000 \mathrm{ml}$ of Ringer's lactate solution over an $8 \mathrm{~h}$ period. Tenoxicam (20 mg) was intravenously administered before extubation.

In the SA group, $2.5 \mathrm{ml}$ of hyperbaric bupivacaine (0.5\%) with $10 \mu \mathrm{g}$ of fentanyl were injected into all patients at the L3-L4 interspace in the sitting position. A 26-gauge spinal needle was used while performing spinal anesthesia. Patients were positioned supine with a $20^{\circ}$ left lateral tilt to minimize aortocaval compression. The level of sensory blockade after spinal anesthesia was assessed with cold and pinprick stimulation tests. All patients received $40 \%$ oxygen by face mask. Oxytocin treatment after delivery was the same as in the GA group.

The birth weights and Apgar scores of the neonates at 1 and 5 min after delivery were assessed by a pediatrician who was unaware of the study. An Apgar score of $<7$ was considered to be abnormal. Hemodynamic parameters of all patients (heart rate 
and systolic, diastolic, and mean blood pressures) were recorded before induction of anesthesia and every $10 \mathrm{~min}$ during the operative procedure. Hypotension was defined as a systolic blood pressure (SBP) of $<100 \mathrm{mmHg}$, and bradycardia was defined as a heart rate of $<60$. Respiratory depression was defined as a decrease in oxygen saturation to $<90 \%$. Immediately after delivery, maternal and fetal blood samples were obtained from veins in the antecubital fossa and the umbilical veins, respectively. After coagulation of blood at room temperature, sera were separated by centrifugation at $2000 \times \mathrm{g}$ for $10 \mathrm{~min}$ at room temperature. All samples were then stored at $-80^{\circ} \mathrm{C}$ until analysis.

\section{Biochemical measurements}

Serum neopterin concentrations were measured with a highperformance liquid chromatography device (1200 Series System; Agilent Technologies, Santa Clara, CA, USA). In brief, $100 \mu \mathrm{l}$ of $100 \%$ acetonitrile was added to $100 \mu \mathrm{l}$ of serum and vortexed. The precipitated protein was removed by centrifugation at $10000 \times \mathrm{g}$ for $10 \mathrm{~min}$ at $4^{\circ} \mathrm{C}$. One hundred microliters of the supernatant were filtered through a $0.2 \mu \mathrm{m}$ filter and then injected into the chromatographic system. Separation of neopterin was achieved with a $150 \times 4.6 \mathrm{~mm}$ I.D. Inertsil ODS-3, C18 RP column with a particle size of $5 \mu \mathrm{m}$ (Hichrom Lim., Berkshire, UK) fitted with a $10 \times 4.6 \mathrm{~mm}$ I.D. Phenomenex ODS-2 guard column (Phenomenex Inc., Torrance, USA) using water/acetonitrile $(99 / 1, \mathrm{v} / \mathrm{v})$ as the mobile phase (isocratic elution) at a flow rate of $1.0 \mathrm{ml} / \mathrm{min}$. The areas of peaks detected by fluorescent detector (Ex, $353 \mathrm{~nm}$; Em, $438 \mathrm{~nm}$ ) were used for quantification. Serum neopterin levels are expressed as nmol/l.

Serum E-selectin and L-selectin concentrations were measured using Human sE-selectin and sL-selectin Instant ELISA kits (Bender MedSystems, Vienna, Austria) according to the manufacturer's instructions. The calculated overall intra-assay and interassay coefficients of variation for sE-selectin were 5.4 and $6.0 \%$, respectively, and those for sL-selectin analyses

Table 1. Maternal and Neonatal Clinical Characteristics

\begin{tabular}{lccc}
\hline & \multicolumn{2}{c}{ Groups } & \\
\cline { 2 - 3 } & $\begin{array}{c}\text { Spinal } \\
\text { anesthesia } \\
(\mathrm{n}=20)\end{array}$ & $\begin{array}{c}\text { General } \\
\text { anesthesia } \\
(\mathrm{n}=20)\end{array}$ & $\mathrm{P}$ \\
\hline Maternal age in years (yr) & $29.7 \pm 5.0$ & $30.2 \pm 5.8$ & 0.751 \\
Primiparae (n, \%) & $7(35)$ & $8(40)$ & 0.782 \\
Gestational age at delivery (week) & $39.1 \pm 0.9$ & $38.5 \pm 1.1$ & 0.061 \\
Birth weight of newborn (g) & $3504 \pm 455$ & $3266 \pm 487$ & 0.119 \\
Apgar score at 1 min & $7(7-9)$ & $7(7-9)$ & 0.968 \\
Apgar score at 5 min & $9(9-10)$ & $9(9-10)$ & 1.000 \\
\hline
\end{tabular}

All data are presented as means \pm standard deviation, medians (minimum-maximum), or frequencies. were 3.7 and $4.2 \%$, respectively. The limits of detection of human sE-selectin and sL-selectin were 0.163 and $0.182 \mathrm{ng} / \mathrm{ml}$, respectively. All standards and samples were measured in duplicate.

Leukocyte counts were performed using an automatic hematology blood analyzer (ABX Pentra DX 120; Horiva ABX Diagnostics, Montpellier, France).

\section{Statistical analysis}

All statistical analyses were performed using the SPSS 15.0 software (SPSS Inc., Chicago, IL, USA). Distributions were evaluated using the one-sample Kolmogorov-Smirnov test to determine whether the continuous variables were normally distributed. The normally distributed variables were compared using Student's t-test, and others were compared using the MannWhitney $U$ test. The results are expressed as means \pm standard deviation, medians (minimum-maximum), or frequencies. Spearman's correlation coefficients were calculated to represent the relationship between maternal and cord blood levels. A P value of $<0.05$ was considered to indicate statistical significance for overall comparisons.

\section{Results}

There were no significant differences in maternal age, gestational age, birth weight of the newborns, or Apgar scores between the two groups (Table 1). The anesthetic technique had no effect on Apgar scores. Apgar scores in both groups were normal (Table 1). The duration of anesthesia, duration of the operation, and the time of baby-out were similar between the two groups (Table 2). No asphyxia or respiratory distress syndrome occurred in any neonates.

Serial SBP and HR values during the operation after the onset of SA and GA are shown in Fig. 1 and 2, respectively. SBP in the SA group was significantly lower than that in the GA group at 1 and 20 min of the operation, but the lowest SBP in the GA group was at $30 \mathrm{~min}$. HR in the SA group was significantly higher than that in the GA group at 1 and $10 \min (\mathrm{P}<0.020$ and $\mathrm{P}<0.009$, respectively). However, HR in the GA group was higher than

Table 2. Operation and Anesthesia Durations

\begin{tabular}{|c|c|c|c|}
\hline & \multicolumn{2}{|c|}{ Groups } & \multirow[b]{2}{*}{$\mathrm{P}$} \\
\hline & $\begin{array}{c}\text { Spinal } \\
\text { anesthesia } \\
(\mathrm{n}=20)\end{array}$ & $\begin{array}{c}\text { General } \\
\text { anesthesia } \\
(\mathrm{n}=20)\end{array}$ & \\
\hline Duration of anesthesia & $38(30-50)$ & $35(35-50)$ & 0.437 \\
\hline Duration of operation & $30(25-40)$ & $30(30-45)$ & 0.286 \\
\hline Time of baby-out & $3(2-4)$ & $3(2-5)$ & 0.353 \\
\hline
\end{tabular}

All data are presented as medians (minimum-maximum) in min. 
that in the SA group at 20 min of the operation. Bradycardia was not observed in any patient in either group. The lowest blood pressure in the SA group occurred at 20 and $30 \mathrm{~min}$ of the operation (Table 3 ). In contrast, there were differences at 1,20 , and $30 \mathrm{~min}$ of the operation in both groups in terms of saturation of peripheral oxygen (Table 3).

There were no differences between the two groups (SA and GA) in leukocyte counts in maternal and cord blood samples

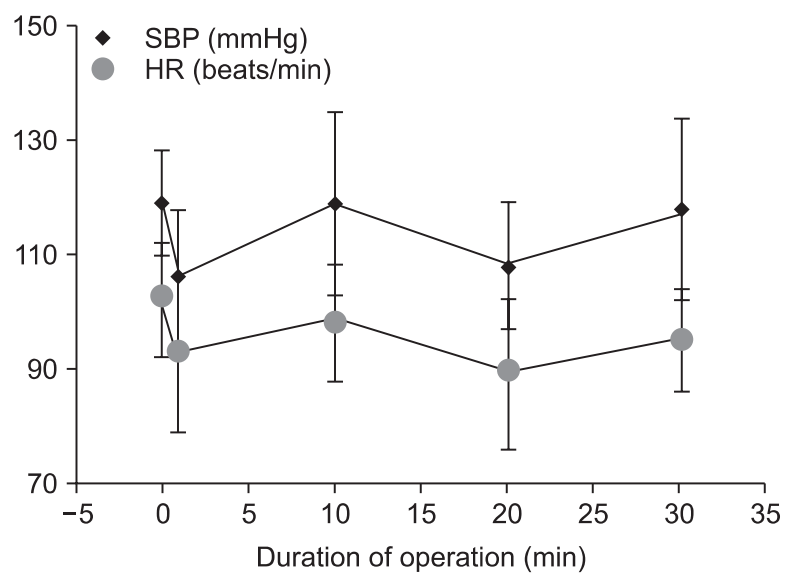

Fig. 1. Hemodynamic differences in the spinal anesthesia group. SBP: systolic blood pressure, HR: heart rate.

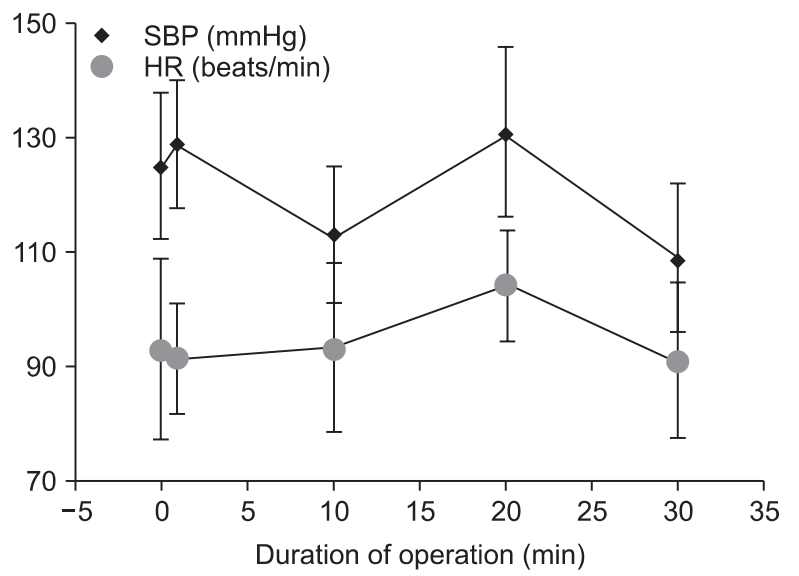

Fig. 2. Hemodynamic differences in the general anesthesia group. SBP: systolic blood pressure, HR: heart rate.
( $\mathrm{P}=0.164$ and $\mathrm{P}=0.745$, respectively) (Table 4$)$.

Cord blood neopterin concentrations in the SA group were not different from those in the GA group, but maternal neopterin levels in the SA group were different from those in the GA group ( $\mathrm{P}=0.465$ and $\mathrm{P}<0.001$, respectively) (Table 4). Maternal and cord blood neopterin concentrations were not correlated in the two groups. However, there was a significant correlation between the maternal sL-selectin and cord blood sL-selectin levels in the GA group ( $\mathrm{r}=488, \mathrm{P}=0.029)$.

The maternal blood levels of sE-selectin and sL-selectin were not different between the SA and GA groups $(\mathrm{P}=0.579$ and $\mathrm{P}=$ 0.725 , respectively) (Table 4). Similarly, the cord blood levels of sE-selectin and sL-selectin were not different from the maternal levels in both the SA and GA groups $(\mathrm{P}=0.273$ and $\mathrm{P}=0.725$, respectively) (Table 4 ). We found an increased inflammatory process in the fetal circulation due to the anesthetic method in both groups. sE-selectin and neopterin levels and leukocyte counts were higher in the fetal circulation than in the maternal circulation in in the SA group $(\mathrm{P}<0.001, \mathrm{P}<0.001$, and $\mathrm{P}=0.001$, respectively). In addition, the $\mathrm{sE}$-selectin and neopterin levels and leukocyte counts were higher in the fetal circulation than in the maternal circulation in the GA group $(\mathrm{P}=0.001, \mathrm{P}<0.001$, and $\mathrm{P}=0.052$, respectively).

Table 3. Hemodynamic Differences between the Two Groups

\begin{tabular}{|c|c|c|c|}
\hline & \multicolumn{2}{|c|}{ Groups } & \multirow[b]{2}{*}{$\mathrm{P}$} \\
\hline & $\begin{array}{c}\text { Spinal } \\
\text { anesthesia } \\
(\mathrm{n}=20)\end{array}$ & $\begin{array}{c}\text { General } \\
\text { anesthesia } \\
(\mathrm{n}=20)\end{array}$ & \\
\hline $\mathrm{MBP}$ at $0 \mathrm{~min}(\mathrm{mmHg})$ & $92(76-110)$ & $97(66-115)$ & 0.144 \\
\hline $\mathrm{MBP}$ at $1 \mathrm{~min}(\mathrm{mmHg})$ & $79(53-98)$ & $104(66-132)$ & $<0.001$ \\
\hline $\mathrm{MBP}$ at $10 \mathrm{~min}(\mathrm{mmHg})$ & $76(51-85)$ & $92(69-113)$ & $<0.001$ \\
\hline $\mathrm{MBP}$ at $20 \mathrm{~min}(\mathrm{mmHg})$ & $68(54-91)$ & $87(60-120)$ & 0.004 \\
\hline $\mathrm{MBP}$ at $30 \mathrm{~min}(\mathrm{mmHg})$ & $72(45-94)$ & $86(58-115)$ & 0.020 \\
\hline $\mathrm{SpO}_{2}$ at $0 \min (\%)$ & $98(96-100)$ & $99(97-100)$ & 0.245 \\
\hline $\mathrm{SpO}_{2}$ at $1 \min (\%)$ & $98(96-100)$ & $99(97-100)$ & 0.013 \\
\hline $\mathrm{SpO}_{2}$ at $10 \min (\%)$ & $98(96-100)$ & $99(97-100)$ & 0.054 \\
\hline $\mathrm{SpO}_{2}$ at $20 \mathrm{~min}(\%)$ & $98(97-100)$ & $99(97-100)$ & 0.039 \\
\hline $\mathrm{SpO}_{2}$ at $30 \mathrm{~min}(\%)$ & $98(96-100)$ & $99(97-100)$ & 0.016 \\
\hline
\end{tabular}

All data are presented as medians (minimum-maximum). MBP: mean blood pressure, $\mathrm{SpO}_{2}$ : saturation of peripheral oxygen.

Table 4. Neopterin, sE-selectin, and sL-selectin Levels and Leukocyte Counts in Maternal and Cord Blood

\begin{tabular}{|c|c|c|c|c|c|c|}
\hline \multirow{2}{*}{ Parameters } & \multicolumn{3}{|c|}{ Maternal blood } & \multicolumn{3}{|c|}{ Umbilical cord blood } \\
\hline & Spinal anesthesia & General anesthesia & $\mathrm{P}$ & Spinal anesthesia & General anesthesia & $\mathrm{P}$ \\
\hline Neopterin (nmol/L) & $4.71(3.77-8.35)$ & $7.06(5.08-12.40)$ & $<0.001$ & $17.7(12.5-28.7)$ & $18.7(1.65-22.4)$ & 0.465 \\
\hline sE-selectin (ng/ml) & $0.37(0.26-0.66)$ & $0.39(0.18-0.61)$ & 0.579 & $1.02(0.69-1.54)$ & $0.97(0.24-2.04)$ & 0.273 \\
\hline sL-selectin (ng/ml) & $0.58(0.24-1.24)$ & $0.53(0.23-1.98)$ & 0.725 & $0.59(0.54-1.15)$ & $0.58(0.43-1.08)$ & 0.725 \\
\hline Leukocyte count $\left(\times 10^{9} / \mathrm{L}\right)$ & $10.7(7.2-13.9)$ & $11.7(6.1-20.1)$ & 0.164 & $13.3(7.2-17.4)$ & $13.3(8.4-19.1)$ & 0.745 \\
\hline
\end{tabular}

All data are presented as medians (minimum-maximum). 


\section{Discussion}

Obstetric anesthesia has various operative risks due to maternal changes. Both regional and GA may cause serious complications [15]. Among pregnant patients, GA is difficult and risky due to the potential for failed endotracheal intubation, postoperative nausea and vomiting, and aspiration of gastric contents [16]. SA is the most commonly used anesthetic technique during CS because of its facility of application, low maternal and fetal morbidity and mortality, and high patient safety [17]. The side effects of each anesthetic technique are often evaluated by maternal hypotension, nausea/vomiting, neonatal Apgar scores, and neonatal acid-base status. The most frequent complication of SA is maternal hypotension. Maternal hypotension may cause impaired placental perfusion and hypoxia in the newborn [16]. In our study, Apgar scores were similar in both groups. The type of anesthesia (SA or GA) had no effect on Apgar scores. SBP in the SA group was significantly lower than that in the GA group at 1 and $20 \mathrm{~min}$ of the operation. Hypotension was observed in a few patients in both groups because of preoperative fluid replacement. SA may cause bradycardia [15]. However, in our study, bradycardia was not observed in either group.

The postoperative neuroendocrine stress response characterized by an increase in the white blood cell count induced by surgery and GA can reportedly be prevented by epidural anesthesia [18]. Local anesthetics inhibit immune function and the migration of leukocytes to the area of inflammation. They have been shown to reduce the release of proinflammatory cytokines [19]. However, the cytokine response during the operation may depend on the degree of tissue injury [5]. De Jongh et al. [20] reported that after CS, IL-6 levels were high at both 12 and $24 \mathrm{~h}$ postoperatively with epidural and GA. Therefore, the cytokine response to CS is not influenced by the anesthetic technique used. Furthermore, the synthesis of cytokines can be adjusted by various hormones such as prostaglandins, estrogens, progesterone, and cortisol [21].

Some studies have reported that the mode of delivery and parturition are associated with an increase in proinflammatory cytokines [22]. Uteroplacental hypoxemia caused by SA may result in inflammatory changes in the placenta and induce the release of proinflammatory cytokines and selectins. These in turn may lead to activation of fetal leukocytes and an increase in the fetal leukocyte count. Extravasation of leukocytes to the area of inflammation is an important feature of inflammation, and this condition also requires cell adhesion molecules. These molecules play significant roles in the recruitment, adhesion, and migration of leukocytes [23]. Moreover, the fetal membranes play an important role in the inflammatory response by increasing the production of proinflammatory cytokines during pregnancy [23]. In addition, molecular changes at the maternal-fetal interface are a pivotal mechanism. All of these events may cause changes in both the maternal and fetal immune systems [24].

In our study, we found that the mean cord blood leukocyte counts and sE-selectin levels were higher than the maternal levels in both groups. In addition, the mean cord blood neopterin levels were higher than the mean maternal blood levels in both groups. These results show that the anesthesia given to the mother during the operation can lead to changes in the fetal immune system. However, there is no significant effect on the levels of sL-selectin in either the maternal or cord blood with either anesthetic method. The synthesis and expression of selectins is rapidly induced by proinflammatory cytokines [25]. Gearing and Newman proposed that sL-selectin levels increase in acute inflammatory conditions [26]. However, we found lower sL-selectin levels due to anesthesia in both maternal and cord blood. This decrease in sL-selectin levels may also be a result of downregulation of leukocyte L-selectin expression [27]. It has also been reported that decreased sL-selectin levels may improve the interaction between endothelial cells and leukocytes [28].

Increased sE-selectin and decreased sL-selectin levels may suggest leukocyte adhesion to endothelial cells. Furthermore, increased leukocyte counts may indicate leukocyte transmigration between tissues. Neutrophil-endothelial cell interaction may be an important factor in the hemodynamic changes at the microvascular interface during anesthesia. We showed increased inflammation in the fetal circulation in both anesthesia groups. However, decreased sL-selectin and sE-selectin levels in cord blood may also reflect prematurity [28]. Biao et al. [29] reported that the inhibition of upregulation of E-selectin by desflurane may be due to reduced activation of sE-selectin and may also inhibit neutrophil adhesion.

Maternal neopterin levels were reportedly higher in pregnant than in non-pregnant females [30]. In our previous study, we found that both maternal and cord blood neopterin levels during normal pregnancy were higher than those in females with pre-eclampsia. We also found no correlation between the neopterin levels of cord and maternal blood between pre-eclampsia and normal pregnancy groups. Conversely, another study reported a significant correlation between maternal and neonatal neopterin concentrations [30]. In the present study, maternal neopterin levels were lower in the SA group than in the GA group. In addition, the neopterin concentrations were higher in the fetal circulation than in the maternal circulation. Neopterin levels are increased during the cell-mediated immune response, which reflects the activation of macrophages/monocytes [30]. In contrast, high cord blood neopterin levels may also be associated with the maturation of the fetal immune system [30].

Our study has some limitations. It had a relatively small sample size and did not include individuals who had a normal birth without anesthesia. We also did not measure the proinflamma- 
tory cytokine levels in the maternal and cord blood samples.

In conclusion, the results of our study indicate that both GA and SA techniques increased the levels of inflammatory cell adhesion molecules, such as sE-selectin and neopterin, in neonates, but did not increase the level of sL-selectin. We did not find a correlation between maternal and fetal inflammatory responses. Further studies are required to investigate the inflammatory response to anesthetic techniques.

\section{References}

1. Ng K, Parsons J, Cyna AM, Middleton P. Spinal versus epidural anaesthesia for caesarean section. Cochrane Database Syst Rev 2004; (2): CD003765.

2. Høgevold HE, Lyberg T, Kähler H, Haug E, Reikerås O. Changes in plasma IL-1beta, TNF-alpha and IL-6 after total hip replacement surgery in general or regional anaesthesia. Cytokine 2000; 12: 1156-9.

3. Douglas RG, Shaw JH. Metabolic response to sepsis and trauma. Br J Surg 1989; 76: 115-22.

4. Naito Y, Tamai S, Shingu K, Shindo K, Matsui T, Segawa H, et al. Responses of plasma adrenocorticotropic hormone, cortisol, and cytokines during and after upper abdominal surgery. Anesthesiology 1992; 77: 426-31.

5. Shimada M, Winchurch RA, Beloucif S, Robotham JL. Effect of anesthesia and surgery on plasma cytokine levels. J Crit Care 1993; 8: 10916.

6. Järvisalo MJ, Juonala M, Raitakari OT. Assessment of inflammatory markers and endothelial function. Curr Opin Clin Nutr Metab Care 2006; 9: 547-52.

7. Hall GM, Desborough JP. Interleukin-6 and the metabolic response to surgery. Br J Anaesth 1992; 69: 337-8.

8. Blankenberg S, Barbaux S, Tiret L. Adhesion molecules and atherosclerosis. Atherosclerosis 2003; 170: 191-203.

9. Kansas GS. Selectins and their ligands: current concepts and controversies. Blood 1996; 88: 3259-87.

10. Newman W, Beall LD, Carson CW, Hunder GG, Graben N, Randhawa ZI, et al. Soluble E-selectin is found in supernatants of activated endothelial cells and is elevated in the serum of patients with septic shock. J Immunol 1993; 150: 644-54.

11. Sood R, Kalloway S, Mast AE, Hillard CJ, Weiler H. Fetomaternal cross talk in the placental vascular bed: control of coagulation by trophoblast cells. Blood 2006; 107: 3173-80.

12. Müller MM, Curtius HC, Herold M, Huber CH. Neopterin in clinical practice. Clin Chim Acta 1991; 201: 1-16.

13. Cakir E, Akgul OE, Aydin I, Cayci T, Kurt YG, Onguru O, et al. The association between neopterin and acetaminophen-induced nephrotoxicity. Ren Fail 2010; 32: 740-6.

14. Coskun O, Oter S, Yaman H, Kilic S, Kurt I, Eyigun CP. Evaluating the validity of serum neopterin and chitotriosidase levels in follow-up brucellosis patients. Intern Med 2010; 49: 1111-8.

15. Jadon A. Complications of regional and general anaesthesia in obstetric practice. Indian J Anaesth 2010; 54: 415-20.

16. Emmett RS, Cyna AM, Andrew M, Simmons SW. Techniques for preventing hypotension during spinal anaesthesia for caesarean section. Cochrane Database Syst Rev 2002; (3): CD002251.

17. Arzola C, Wieczorek PM. Efficacy of low-dose bupivacaine in spinal anaesthesia for Caesarean delivery: systematic review and metaanalysis. Br J Anaesth 2011; 107: 308-18.

18. Volk T, Schenk M, Voigt K, Tohtz S, Putzier M, Kox WJ. Postoperative epidural anesthesia preserves lymphocyte, but not monocyte, immune function after major spine surgery. Anesth Analg 2004; 98: 1086-92.

19. Carvalho B, Clark DJ, Yeomans DC, Angst MS. Continuous subcutaneous instillation of bupivacaine compared to saline reduces interleukin 10 and increases substance P in surgical wounds after cesarean delivery. Anesth Analg 2010; 111: 1452-9.

20. De Jongh RF, Bosmans EP, Puylaert MJ, Ombelet WU, Vandeput HJ, Berghmans RA. The influence of anaesthetic techniques and type of delivery on peripartum serum interleukin-6 concentrations. Acta Anaesthesiol Scand 1997; 41: 853-60.

21. De Jongh RF, Puylaert M, Bosmans E, Ombelet W, Maes M, Heylen R. The fetomaternal dependency of cord blood interleukin-6. Am J Perinatol 1999; 16: 121-8.

22. Gyarmati B, Beko G, Szalay B, Cseh A, Vásárhelyi B, Treszl A. Maternal cytokine balance on the third postpartum day is not affected by the mode of delivery after healthy pregnancies. J Int Med Res 2010; 38: 208-13.

23. Osman I, Crawford M, Jordan F, Young A, Norman J, Thomson A. Expression and localization of cell adhesion molecules in human fetal membranes during parturition. J Reprod Immunol 2004; 63: 11-21.

24. Wicherek L, Galazka K. The possible correlation between the patient's immune tolerance level during cesaerean section and the incidence of subsequent emergency peripartum hysterectomy. Clin Dev Immunol 2007; 2007: 63596.

25. Catarino C, Santos-Silva A, Belo L, Rocha-Pereira P, Rocha S, Patrício B, et al. Inflammatory disturbances in preeclampsia: relationship between maternal and umbilical cord blood. J Pregnancy 2012; 2012: 684384.

26. Gearing AJ, Newman W. Circulating adhesion molecules in disease. Immunol Today 1993; 14: 506-12.

27. Haught WH, Mansour M, Rothlein R, Kishimoto TK, Mainolfi EA, Hendricks JB, et al. Alterations in circulating intercellular adhesion molecule-1 and L-selectin: further evidence for chronic inflammation in ischemic heart disease. Am Heart J 1996; 132: 1-8. 
28. Mellembakken JR, Aukrust P, Hestdal K, Ueland T, Abyholm T, Videm V. Chemokines and leukocyte activation in the fetal circulation during preeclampsia. Hypertension 2001; 38: 394-8.

29. Biao Z, Zhanggang X, Hao J, Changhong M, Jing C. The in vitro effect of desflurane preconditioning on endothelial adhesion molecules and mRNA expression. Anesth Analg 2005; 100: 1007-13.

30. Hammerer-Lercher A, Puschendorf B, Fuchs D, Mair J, Tews G, Shebl O, et al. Possible placental exchange of neopterin as indicated by significant correlations in matched maternal neonatal blood samples at delivery. Clin Chim Acta 2006; 365: 350-1. 